\title{
Application of Cryogenic-Electron Microscopy (Cryo-EM) for Morphological Characterization of Complex Drug Products
}

\author{
Jiwen Zheng ${ }^{1 *}$, Yong $\mathrm{Wu}^{1}$ \\ 1. Division of Biology, Chemistry and Materials Science, Office of Science and Engineering \\ Laboratories, Center for Devices and Radiological Health, Food and Drug Administration, Silver Spring, \\ Maryland, United States \\ * Corresponding author: Jiwen.zheng@fda.hhs.gov
}

A variety of complex drug formulations including emulsions, suspensions, and liposomes have been developed to enhance the bioavailability of active pharmaceutical ingredients (APIs). The complex nature of these formulations presents significant challenges to the development and approval of generic drug products.

Subtle differences in manufacturing processes cause noticeable differences in morphological characteristics of products that are formulated qualitatively (Q1) and quantitatively (Q2) the same. These morphological differences may impact the drug release behavior and bioavailability of APIs. Therefore, a comparative morphology should be included as a part of physicochemical characterization studies used to demonstrate product sameness. In order to fulfill this demand on demonstrating comparable morphology, a reliable and suitable characterization techniques need to be identified, developed and validated. In this study, cryo-electron microscopy (Cryo-EM) was evaluated as a potential regulatory tool for the morphological characterization of various complex drug products to support demonstration of product sameness.

A wide variety of complex drug products including intravenous iron injections, ophthalmic emulsions, anesthetic emulsion/liposome injections, anticancer liposomal injections and protein-bound paclitaxel nanoparticles were investigated using Cryo-EM $[1,2,3]$. The extracted data indicates useful information such as the globule/particle size distribution, shape, internal structure, agglomeration tendency and surface features. For example, under cryo-TEM, intravenous iron formulations (sodium ferric gluconate, iron sucrose, low molecular weight iron dextran and ferumoxytol) exhibited a narrow particle size distribution, with an averaged iron core size of $2 \mathrm{~nm}$ for all four intravenous iron products tested. Compared to traditional room temperature sample preparation, Cryo-TEM did not show artifacts of agglomeration for the intravenous iron nanoparticles. In addition, Cryo-TEM also provides evidence of oil droplets and lipid vesicles coexisting in an anesthetic injection product allowing one to observe the composition of complex oil and lipid structures reinforcing the idea of variability between approved products. As conventional electron microscopy is inherently associated with various degrees of artifacts during sample preparation, Cryo-EM presents a method for reliable visualization of drug products at the nanoscale, facilitating both the development and regulatory review of generic complex nanoscale drug products.

\section{References:}

[1] Y Wu et al., Int. J. Pharm. 505 (2016), p. 167.

[2] PE Petrochenko et al., Int. J. Pharm. 550 (2018), p. 229.

[3] S Manna et al., J Control Release. 294 (2019), p. 279. 

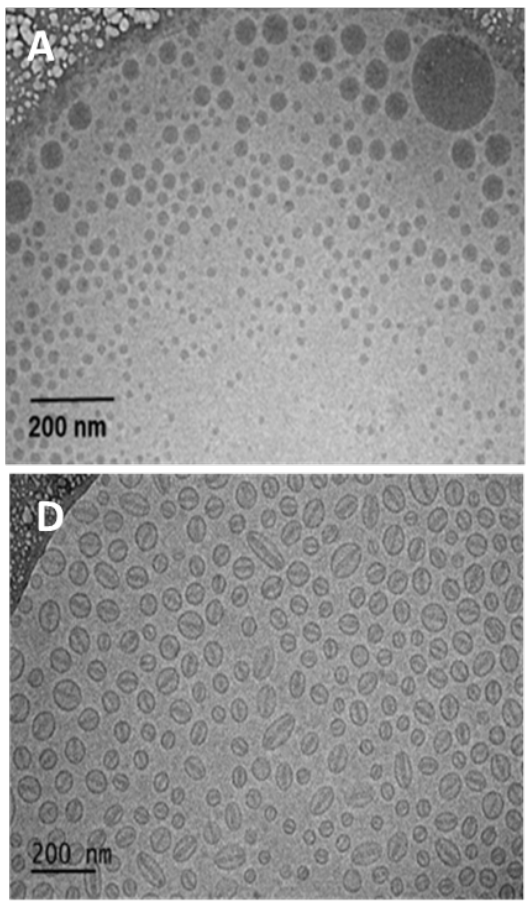
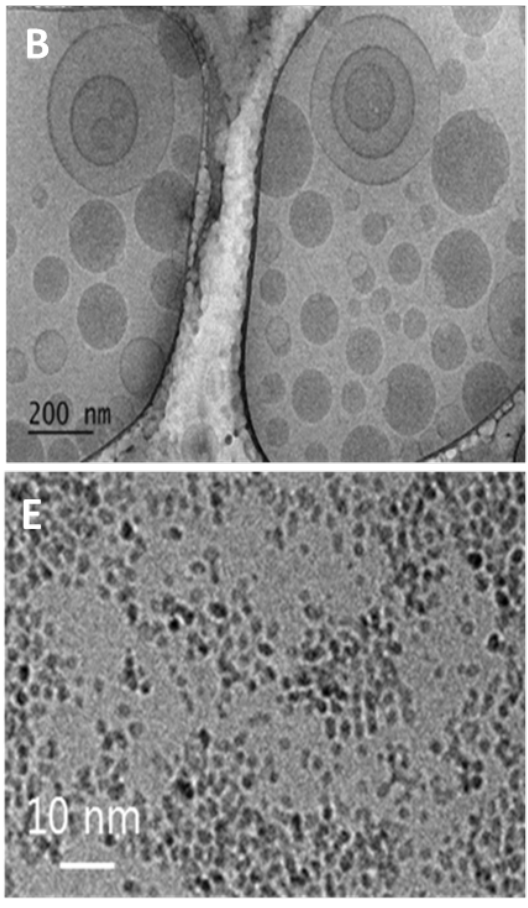
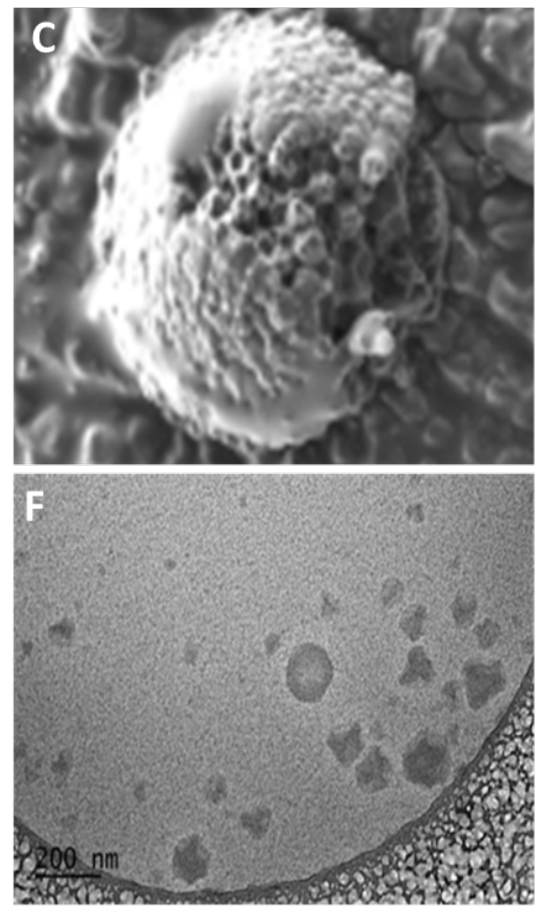

Figure 1. cryo-EM images of (A) cyclosporine ophthalmic emulsion (B) propofol nanoemulsion/liposome (C) multi-vesicular liposome (D) doxorubicin liposome (E) iron dextran complex and (F) albumin-bound paclitaxel nanoparticles 